\title{
O USO DA SIMULAÇÃO NA ANÁLISE DE CENÁRIOS EM OPERAÇÕES DE CARREGAMENTO E TRANSPORTE DA MINERAÇÃO USIMINAS S.A.*
}

Ricardo Melo Pereira ${ }^{1}$

\section{Resumo}

Este trabalho tem como objetivo avaliar modelos de simulação, construídos através do software Talpac® em uma mina a céu aberto, a fim de analisar através de distribuições de probabilidade com o uso do software Minitab®, alguns parâmetros que influenciam diretamente a produtividade dos caminhões fora de estrada. Dentre estes parâmetros, os principais são: Carga média dos caminhões, velocidade média, número de viagens por hora de trabalho e tempo médio de fila na carga. Ao final, os dados de produtividade dos caminhões obtidos no modelo, serão comparados aos dados reais da mina, obtidos através do sistema de gerenciamento de frota Smartmine ${ }^{\circledR}$, podendo este modelo ser validado ou não, tanto em termos de produtividade quanto dos demais parâmetros.

Palavras-chave: Modelo; Simulação; Software; Cenários; Parâmetros; Produtividade.

\section{THE USE OF SIMULATION IN THE SCENARIO ANALYSIS IN LOADING AND TRANSPORT OPERATIONS AT MINERAÇÃO USIMINAS S.A.}

\section{Abstract}

This study aims to evaluate simulation models built by Talpac $®$ software in an open pit mine in order to parse through probability distributions using the Minitab® software, some parameters that directly influence the productivity of trucks off road. Among the parameters, the main ones are: Average load of trucks, average speed, number of cycles per hour worked and average queue load. At the end, the productivity data from the model will be compared to the actual data of the mine, obtained by SmartMine ${ }^{\circledR}$ fleet management system, and this model is validated or not, both in terms of productivity and all other parameters.

Keywords: Mode; Simulation; Software; Settings; Parameters; Productivity. 


\section{INTRODUÇÃO}

A complexidade dos processos, desde a produção à administração, há muito ultrapassou os limites da experiência dos administradores e das ferramentas rotineiras de apoio à tomada de decisão.

Hoje, a principal ferramenta de tomada de decisão em algumas empresas ainda é a planilha eletrônica. Apesar de muito útil e até bastante poderosa, essa ferramenta não é apropriada para estudar processos acima de certa complexidade.

Para decidir sem erro e extrair o máximo dos recursos no sistema, é imprescindível recorrer às ferramentas e técnicas apropriadas. Ferramentas analíticas de planejamento, com simulação e otimização, estão cada vez mais presentes nos modernos processos de tomada de decisão.

Simulando, avaliamos estratégias e diversas maneiras de combinar essas técnicas em diferentes situações de negócio permitem a obtenção de planejamentos mais efi cientes e de menor risco, frente ao enorme universo de decisões alternativas e informações comportamentais dos eventos que compõem as operações de carregamento e transporte de materiais em mineração.

O objetivo deste trabalho é mostrar que, com o auxílio de softwares especializados, podemos desenvolver modelos de simulação que possam auxiliar as equipes de engenharia da Mineração Usiminas S.A na tomada de decisão em relação às operações de carregamento e transporte de materiais na mina, decisões estas relacionadas ao aumento de produtividade da frota de equipamentos, dimensionamento, avaliações econômicas, identificação dos gargalos operacionais, assim como, verificação da exequibilidade dos planos de lavra de curto e médio prazo e elaboração de orçamentos para novos investimentos em equipamentos de carga e transporte.

Em sua essência, a simulação de mineração é um método barato, sem riscos de testar qualquer coisa, desde revisões simples para completar os projetos, sempre com a finalidade de atender as metas de produção com o menor custo possível.

E ao contrário de análise baseada em planilhas e previsão, simulação oferece um meio rápido e eficiente para ajustar parâmetros e simular novamente, economizando tempo e acelerando resultados.

$\mathrm{Na}$ mineração brasileira, a simulação ainda tem o seu uso restrito a poucos trabalhos em grandes empresas e realizados apenas por profissionais especializados.

\section{METODOLOGIA}

\subsection{Identificação e Caracterização do Problema}

O problema diz respeito a se tentar conseguir uma maior previsibilidade e agilidade na tomada de decisões com relação às operações de carregamento e transporte a céu aberto na Mineração Usiminas S.A, que sempre era executado através apenas

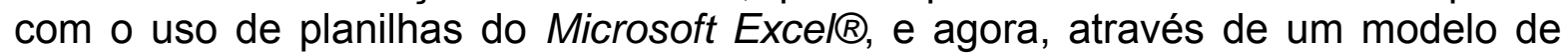
simulação, representando uma de suas duas unidades operacionais, denominada Mina Oeste, utilizando para tal fim, o software Talpac ${ }^{\circledR}$ versão 10 . Inseridos no contexto do problema estão quatro frentes de lavra onde ocorrem as atividades de carregamento e transporte dos caminhões com minério ou estéril, 14 Caminhões fora de estrada modelo Randon Perlini RDP 490 e 2 Escavadeiras front shovel modelo Liebherr R9250. 


\subsection{Coletas de Dados}

Os dados utilizados na construção e implementação do modelo dizem respeito à quantidade, capacidades e tipos de equipamentos, tempos de duração das diversas atividades, índices de utilização e disponibilidade dos mesmos, são dados reais operacionais do primeiro trimestre do ano de 2014, foram extraídos do banco de dados da Mineração Usiminas S.A do software de gerenciamento de frota Smartmine ${ }^{\circledR}$, fabricado pela empresa DEVEX.

\subsection{Análises de Dados}

Após a coleta e organização dos dados em forma de tabelas, foram geradas as distribuições estatísticas para os parâmetros de operação atribuídos aos caminhões, que julgamos mais relevantes, São elas: Produtividade global dos caminhões $(\mathrm{t} / \mathrm{h})$, Carga média das viagens (t/viagem), Velocidade média cheio $(\mathrm{Km} / \mathrm{h})$, Velocidade média vazio $(\mathrm{Km} / \mathrm{h})$, Número de viagens por hora (viagens/h) e Tempo médio de fila na praça de carga (min/viagem). Todas as curvas de distribuição e análises estatísticas referentes aos dados reais foram realizadas com a utilização do software Minitab 16®.

\subsection{Construção e Validação do Modelo}

O modelo foi construído baseado nas premissas mais próximas possíveis da realidade das operações. São elas: Escala de trabalho: 24 h/dia, 7 dias por semana;Velocidade máxima: Limitado a $40 \mathrm{Km} / \mathrm{h}$ em rampas ascendentes e trechos planos; Limitado a $20 \mathrm{Km} / \mathrm{h}$ em rampas descendentes;Equipamento de carga: Escavadeira Liebherr Front Shovel com caçamba de $15 \mathrm{~m}^{3}$; Equipamento de transporte: Caminhão Perlini rígido com caçamba de 95 toneladas líquidas de carga;Metodologia de carga:Priorizar o caminhão cheio (full truck); Manobra simples (single sided truck);Dados operacionais transporte: Spot time at loader (Tempo de manobra na carga) $=30$ segundos;Spot time at dump (Tempo de manobra na descarga $)=30$ segundos; Dumping (Tempo de basculamento $)=60$ segundos $;$ Fator de empolamento: $\mathrm{ROM}=1,33$; Estéril $=1,47$; Densidade do material: $\mathrm{ROM}=2,8$ $\mathrm{t} / \mathrm{m}^{3}$; Estéril $=2,2 \mathrm{t} / \mathrm{m}^{3}$.

Após o modelo ser verificado em forma de programação, ou seja, sem erros de lógica, e configurado de acordo com as características desejadas, o projeto foi finalizado através das simulações de cenários operacionais comparando com parâmetros reais de produção alcançados nas mesmas frentes de lavra, no mesmo período e com a utilização do mesmo porte de equipamentos de carga e transporte utilizados nas operações reais.

\section{ESTUDO DE CASO}

A fim de resumir a apresentação do estudo, dos quatro cenários simulados, será demonstrado em detalhes apenas o Cenário I, este, representativo de todos os cálculos efetuados na simulação.

Cenário I: Frente de lavra: Venus x ITM Oeste; Material: ROM 


\subsection{Produtividades Médias Simuladas $=382,9 \mathrm{t} / \mathrm{h}$ Produtividades Médias Realizadas $=405,0 \mathrm{t} / \mathrm{h}$}

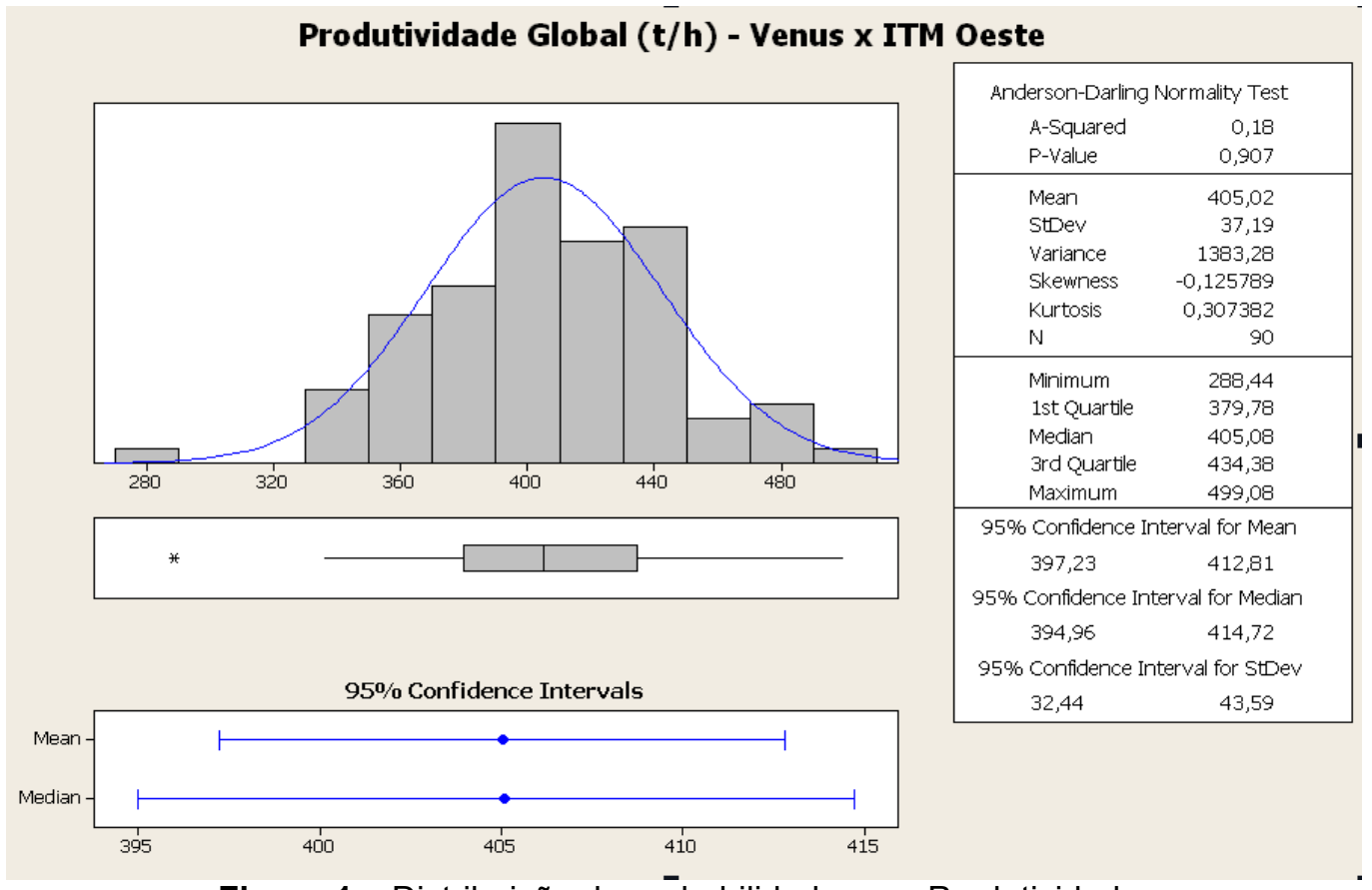

Figura 1 - Distribuição de prōbabilidade para Produtividade.

De acordo com a figura 1 a distribuição estatística de probabilidade para o parâmetro Produtividade do Cenário I é uma curva normal com média 405,0 t/h e desvio padrão 37,1 com $95 \%$ de confiança.

Desvio de apenas 5,4 \% em relação à simulação, ou seja, um ótimo resultado comparativo entre modelo de simulação e operação real para o parâmetro Produtividade nesta frente de lavra.

\subsection{Cargas Médias Simuladas = 94,3 t/v; Cargas Médias Realizadas = 99,6 t/v}

Carga Média (t) - Venus x ITM Oeste
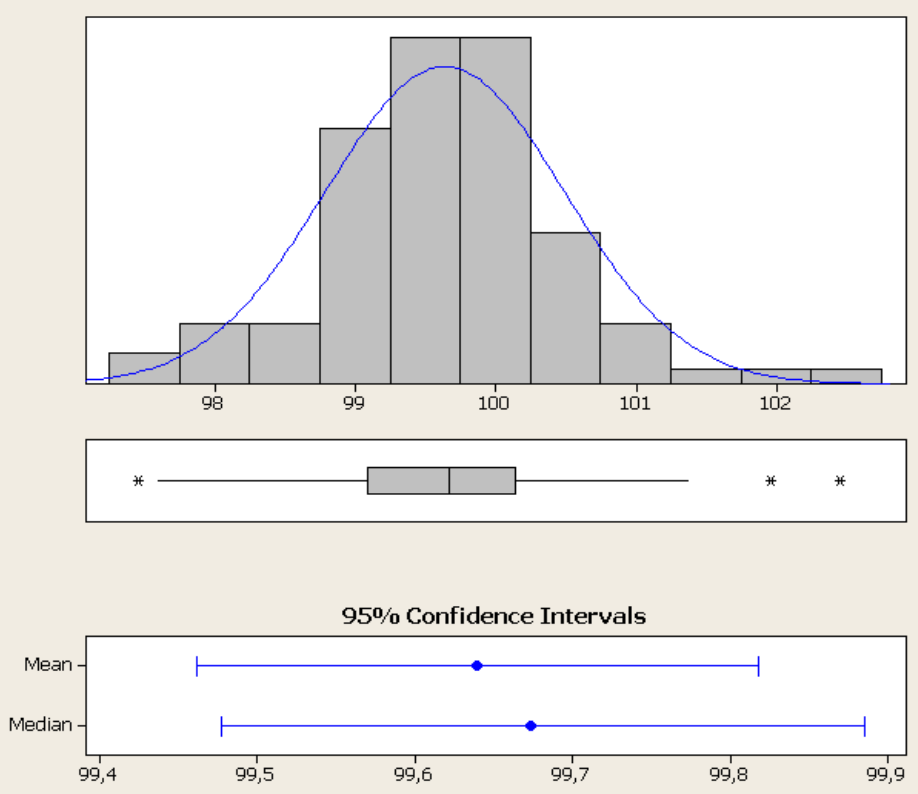

\begin{tabular}{|cc|}
\hline \multicolumn{3}{|c|}{ Anderson-Darling Normality Test } \\
A-Squared & 0,65 \\
P-Value & 0,085 \\
\hline Mean & 99,640 \\
StDev & 0,852 \\
Variance & 0,725 \\
Skewness & 0,17610 \\
Kurtosis & 1,39299 \\
N & 90 \\
\hline Minimum & 97,456 \\
1st Quartile & 99,091 \\
Median & 99,673 \\
3rd Quartile & 100,147 \\
Maximum & 102,459 \\
\hline $95 \%$ Confidence Interval for Mean \\
99,462 & 99,818 \\
$95 \%$ Confidence Interval for Median \\
99,478 & 99,885 \\
$95 \%$ Confidence Interval for StDev \\
0,743 & 0,998 \\
\hline \multicolumn{2}{c}{}
\end{tabular}

Figura 2 - Distribuição de probabilidade para Carga Média 
Para o parâmetro Carga Média, na figura 2, a distribuição estatística de probabilidade também é uma curva normal com média 99,6 t e desvio padrão 0,85. Desvio de apenas $5,3 \%$ em relação à simulação, ou seja, também um ótimo resultado comparativo entre modelo de simulação e operação real para o parâmetro carga média.

\subsection{Velocidades Médias Simuladas $=18,6 \mathrm{Km} / \mathrm{h} ;$ Velocidades Médias Realizadas $=20,7 \mathrm{Km} / \mathrm{h}$}

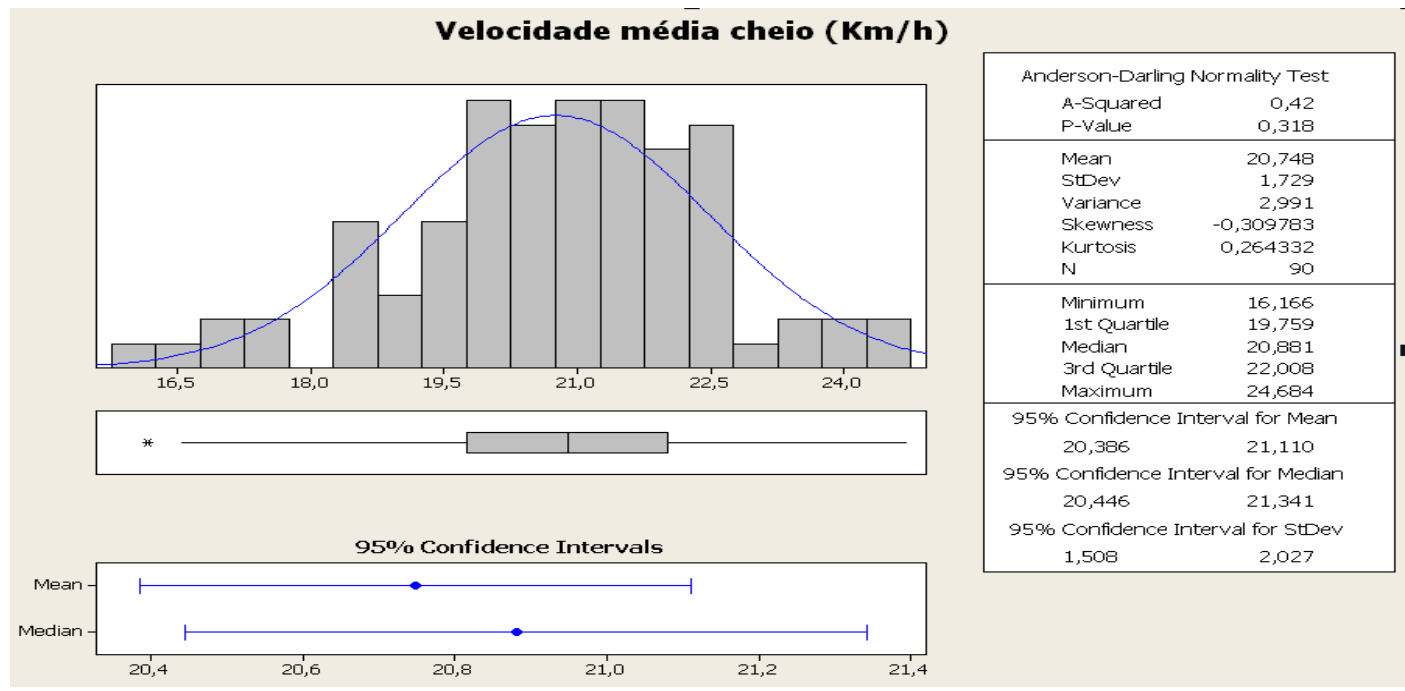

Figura 3 - Distribuição de probabilidade para Velocidade Média Cheio.

Para o parâmetro Velocidade Média Cheio, figura 3, a distribuição estatística de probabilidade é uma curva normal com média $20,7 \mathrm{Km} / \mathrm{h}$ e desvio padrão 1,7.

Velocidade realizada maior do que a simulada, ou seja, é um bom parâmetro comparativo, pois a diferença é muito pequena, apenas $2,1 \mathrm{~km} / \mathrm{h}$ em média por cada ciclo.

\subsection{Velocidade Média Simulada $=25,4 \mathrm{Km} / \mathrm{h}$; Velocidade Média Realizada $=$ $19,0 \mathrm{Km} / \mathrm{h}$}

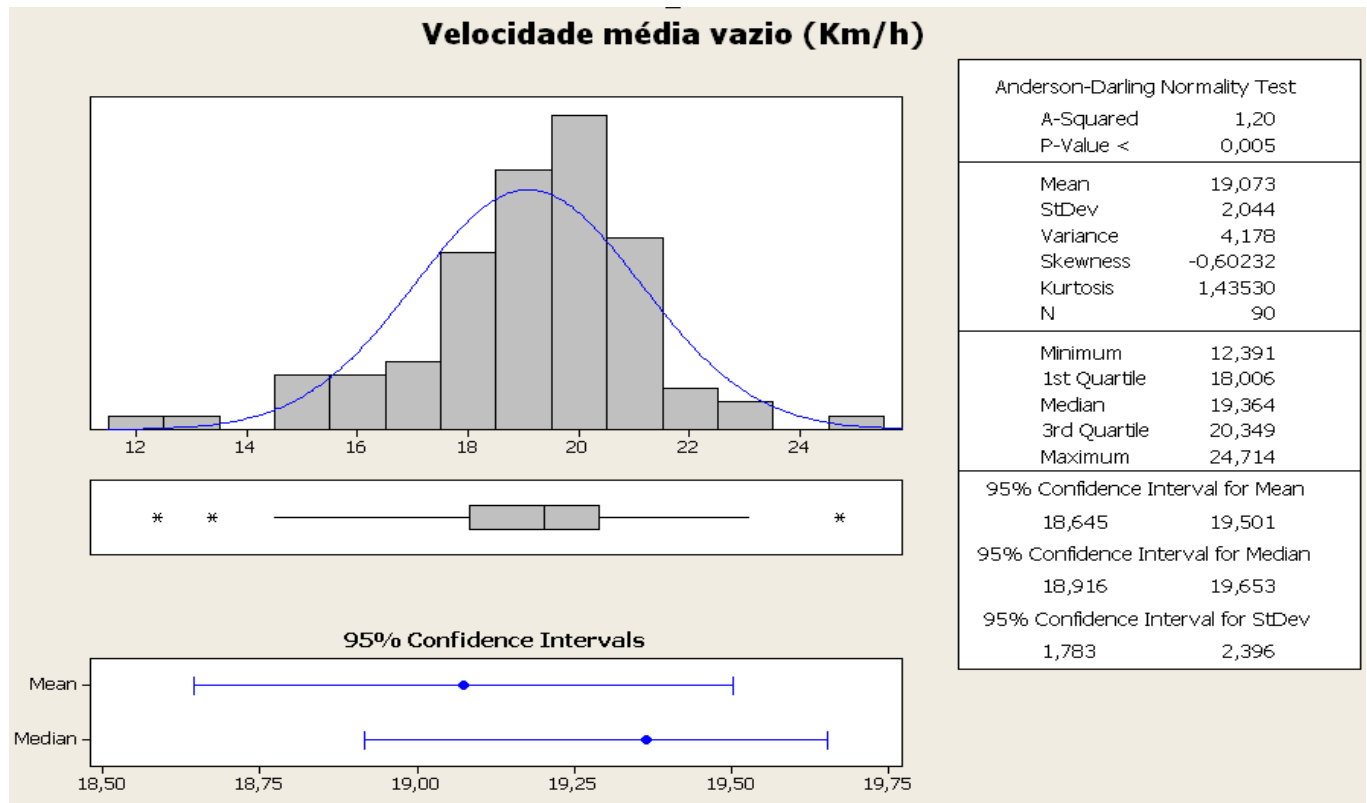

Figura 4 - Distribuição de probabilidade para Velocidade Média Vazio. 
Algumas das causas são conhecidas para diferenças de velocidades entre o real e a simulação, tais como:

O software não considera estreitamentos de pista que às vezes ocorrem na operação real e fazem com que os caminhões diminuam a velocidade.

As irregularidades da pista como depressões e elevações também não são considerados, na interpretação do software a pista de rolamento é isenta dessas imperfeições.

Os raios de curvatura reais não foram realmente medidos em campo antes de serem adaptados ao modelo, apenas estimados.

Não são consideradas interferências com outros veículos e equipamentos que também fazem com que os caminhões diminuam a velocidade, entre outras.

Porém, as mesmas causas que ocorrem quando o caminhão está se deslocando cheio, ocorrem quando ele está vazio. Não foi detectada a causa de uma velocidade ter maior diferença que a outra entre modelo e realidade operacional.

Ou seja, no parâmetro velocidade média vazio, figura 4, dos caminhões, o software, da maneira como foi modelado para este estudo, ainda não está sendo um bom comparativo à realidade operacional.

\subsection{Viagens por hora Simulada $=4,1 \mathrm{v} / \mathrm{h}$; Viagens por hora Realizada $=4,0 \mathrm{v} / \mathrm{h}$}

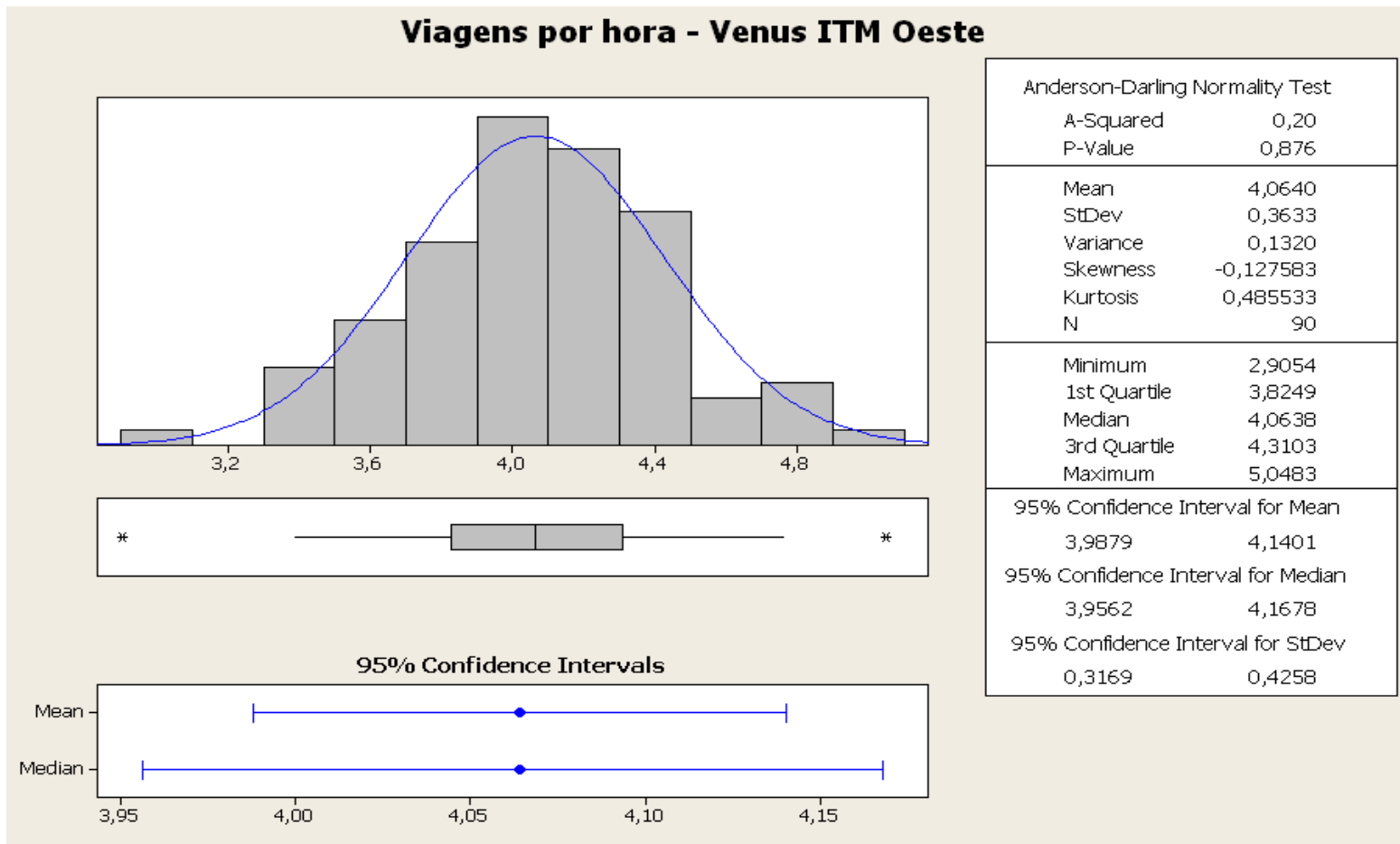

Figura 5 - Distribuição de probabilidade para Viagens por hora.

De acordo com a figura 5 a distribuição estatística de probabilidade para o parâmetro Viagens por hora do Cenário I é uma curva normal com média 4,0 v/h e desvio padrão 0,36 com $95 \%$ de confiança.

Desvio de apenas $2 \%$ em relação à simulação, ou seja, um ótimo resultado comparativo entre modelo de simulação e operação real para o parâmetro Número de Viagens por hora. 


\subsection{Tempos Médios de Fila Simulada = 0,34 $\mathrm{min} /$ ciclo; Tempo Médios de Fila Realizada $=1,0 \mathrm{~min} /$ ciclo}

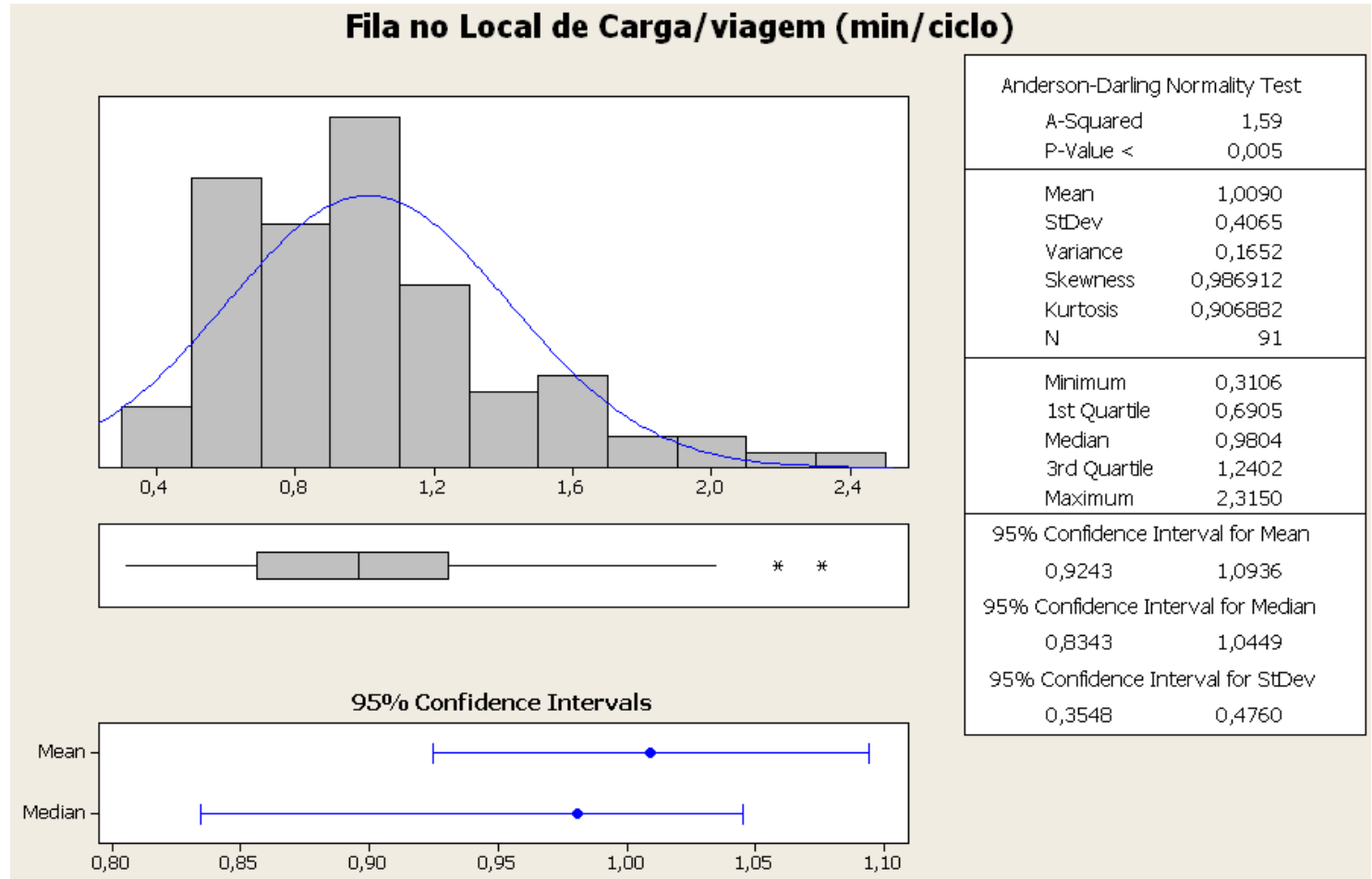

Figura 6 - Distribuição de probabilidade para Fila na carga / viagem.

$66 \%$ acima do tempo de fila real em relação à simulação, para o parâmetro Fila na carga / Viagem (Figura 6).

A explicação está no tempo de carregamento da simulação, muito alto em relação ao tempo em que foi realizado, gerando fila de caminhões na praça de carga.

\section{CONCLUSÃO}

Após, simulados quatro cenários de carregamento e transporte das operações da Mineração Usiminas S.A, os parâmetros ou índices operacionais, que afetam diretamente o índice Produtividade global dos caminhões, foram analisados e agrupados, e além destes, um dos mais importantes, a DMT, foi considerada e descrita na análise final comparativa.

A respeito do Cenário I em uma frente de carregamento e transporte de ROM ou minério, podemos concluir que a diferença apresentada entre produtividade simulada e real é aceitável, $5 \%$.

O software Talpac ${ }^{\circledR}$ atende aos objetivos propostos de comparativo dos modelos com a realidade operacional da Mineração Usiminas S.A, se mostrando uma boa ferramenta para utilização em análises de novas aquisições de equipamentos, elaboração de metas, verificação de exequibilidade dos planos de produção, projeção de planos futuros e orçamentos, porém é preciso um aprimoramento dos estudos em relação ao parâmetro velocidade a fim de ser mais bem modelado e, quando comparado à realidade das operações, conseguirmos resultados satisfatórios, ou seja, muito próximos ou iguais ao realizado em campo.

Nos próximos modelamentos, serão corretamente acrescidos os verdadeiros raios de curvatura, inferência do "fator operador" como causa de perdas e comparação 
das velocidades em campo por trechos menores, a fim de termos uma maior aproximação do real.

\section{BIBLIOGRAFIA}

1 Costa FP. Um modelo de programação matemática para alocação estática de caminhões visando ao atendimento de metas de produção e qualidade. REM: Revista Escola de Minas. Ouro Preto. 2005. p. 77-81.

2 Martins AG. Simulação das operações de lavra da Mina de Brucutu utilizando um modelo de programação linear para alocar os equipamentos de carregamento Dissertação de Mestrado. Programa de Pós-Graduação em Engenharia Mineral da Escola de Minas da Universidade Federal de Ouro Preto. 2013.

3 Meyer MF. Análise de cenários envolvendo operações de carregamento e transporte em uma mina a céu aberto utilizando simulação. Dissertação de Mestrado. Programa de Pós-Graduação em Engenharia Mineral da Escola de Minas da Universidade Federal de Ouro Preto. 2002. 100 p.

4 Pantuza JRG, Souza MJF. Um modelo de simulação no ARENA para o sequenciamento e redução do tempo das viagens dos caminhões em uma mina a céu aberto. XVIII Simpósio de Engenharia de Produção - SIMPEP. 2011. 13 p.

5 Prado DS. Usando o Arena em simulação. Série Pesquisa Operacional. Vol. 3 INDG. 2010.

6 Ramos NAN, Pinto LR. Template do programa ARENA para simulação das operações de carregamento e transporte em mina a céu aberto. REM: R. ESC. MINAS, Ouro Preto, 57 (1): 65-69, jan. mar. 2004. 52. 